\title{
Calculation of $\rho$ meson decay width from the PACS-CS configurations
}

\section{PACS-CS collaboration:}

S. Aoki ${ }^{a, b}$, K.-I. Ishikawa $^{c}$, N. Ishizuka ${ }^{* a, b}$, K. Kanaya $^{a}$, Y. Kuramashi ${ }^{a, b}$, Y. Namekawa ${ }^{b}$, M. Okawa $^{c}$, Y. Taniguchi ${ }^{a, b}$, A. Ukawa ${ }^{a, b}, \mathbf{N}$. Ukita ${ }^{b}$, T, Yamazaki ${ }^{b}$, T. Yoshié ${ }^{a, b}$

${ }^{a}$ Graduate School of Pure and Applied Sciences, University of Tsukuba, Tsukuba 305-8571, Japan.

${ }^{b}$ Center for Computational Sciences, University of Tsukuba, Tsukuba 305-8577, Japan.

${ }^{c}$ Department of Physics, Hiroshima University, Higashi-Hiroshima 739-8526, Japan.

We present preliminary results on the $\rho$ meson decay width from $N_{f}=2+1$ full QCD configurations generated by PACS-CS Collaboration. The decay width is estimated from the $P$-wave scattering phase shift for the isospin $I=1$ two-pion system. The finite size formula presented by Lüscher in the center of mass frame and its extension to non-zero total momentum frame by Rummukainen and Gottlieb are employed for the calculations of the phase shift. Our calculations are carried out at $m_{\pi}=410 \mathrm{MeV}\left(m_{\pi} / m_{\rho}=0.46\right)$ and $a=0.091 \mathrm{fm}$ on a $32^{3} \times 64(L a=2.9 \mathrm{fm})$ lattice.

The XXVIII International Symposium on Lattice Field Theory,

Lattice 2010

June 14-19, 2010

Villasimius, Italy

\footnotetext{
*Speaker.

†E-mail : ishizuka@ccs.tsukuba.ac.jp
} 


\section{Introduction}

Study of the $\rho$ meson decay is a significant step for understanding the dynamical aspects of hadron interactions with lattice QCD. In the early stage of studies toward this direction the transition amplitude $\langle\rho \mid \pi \pi\rangle$ extracted from the time behavior of the correlation function $\langle\pi(t) \pi(t) \rho(0)\rangle$ has been used to estimate the decay width, assuming that the hadron interaction is small $[1,2,3,4]$.

A more realistic approach is an estimation of the decay width from the $P$-wave scattering phase shift for the isospin $I=1$ two-pion system. The finite size formula presented by Lüscher in the center of mass frame [5] or its extension to non-zero total momentum frame by Rummukainen and Gottlieb [7] is employed for an estimation of the phase shift. The first study of this approach was carried by CP-PACS Collaboration using $N_{f}=2$ full QCD configurations $\left(m_{\pi}=330 \mathrm{MeV}, a=\right.$ $0.21 \mathrm{fm}, L a=2.5 \mathrm{fm}$ ) [7]. After this work, two studies were reported with $N_{f}=2$ configurations on finer lattices, one by QCD-SF Collaboration $\left(m_{\pi}=240-810 \mathrm{MeV}, a=0.072-0.084 \mathrm{fm}\right)$ [8] and the other by ETMC Collaboration $\left(m_{\pi}=391 \mathrm{MeV}, a=0.086 \mathrm{fm}, L a=2.1 \mathrm{fm}\right)$ [9].

In the present work we extend these studies by employing $N_{f}=2+1$ full QCD configurations and working on a larger lattice volume. Our calculations are carried out on a subset of configurations previously generated by PACS-CS Collaboration with the Iwasaki gauge action and nonperturbatively $O(a)$-improved Wilson fermion action at $\beta=1.9$. on a $32^{3} \times 64$ lattice [10]. The subset coresponds to the hopping parameters $\kappa_{u d}=0.13754$ for the up and down quark and $\kappa_{s}=0.13640$ for the strange quark. The parameters determined from the spectrum analysis for this subset are $m_{\pi}=410 \mathrm{MeV}\left(m_{\pi} / m_{\rho}=0.46\right), a=0.091 \mathrm{fm}$ and $L a=2.91 \mathrm{fm}$. All calculations are carried out on the PACS-CS computer at Center for Computational Sciences, University of Tsukuba. We note that ETMC [11] and BMW [12] Collaborations reported their preliminary results at Lattice 2010.

\section{Method}

We consider the center of mass frame $(\mathrm{CM})$ and the non-zero total momentum frame (the moving frame (MF)) with the total momentum $\mathbf{p}=(2 \pi / L) \mathbf{e}_{3}$. In these frames the ground $(n=1)$ and the first exited states $(n=2)$ with spin $J=1$ and isospin $I=1$, ignoring hadron interactions, are given by

$$
\begin{array}{lcllll}
\text { frame } & \mathbf{p} L /(2 \pi) & \mathrm{g} & \Gamma & n=1 & n=2 \\
\mathrm{CM} & (0,0,0) & O_{h} & \mathbf{T}_{1}^{-} & \varrho_{j}(\mathbf{0}) & \pi\left(\mathbf{e}_{j}\right) \pi\left(-\mathbf{e}_{j}\right)[1.3] \\
\text { MF } & (0,0,1) & D_{4 h} & \mathbf{E}^{-} & \underline{\rho_{1,2}(0,0,1)} & \pi(1,0,1) \pi(-1,0,0), \pi(0,1,1) \pi(0,-1,0)[1.4] \\
\text { MF } & (0,0,1) & D_{4 h} & \mathbf{A}_{2}^{-} & \underline{\rho_{3}(0,0,1)} & \underline{\pi(0,0,1) \pi(0,0,0)[1.02]}
\end{array}
$$

where $\mathbf{p}$ is the total momentum, $\mathrm{g}$ is the rotational group on the lattice and $\Gamma$ is the irreducible representation of the group. The vectors in parentheses after $\pi$ and $\rho$ refer to the momenta of the pion and the $\rho$ meson in units of $2 \pi / L$. The numbers in square brackets of the two-pion states are values of $\sqrt{s} / m_{\rho}$ on our full QCD configurations. In the present work we calculate the scattering phase shifts of the states marked by under-bar in (2.1). The finite size formulas for these states are given in Refs. [5, 6].

For the $\mathbf{T}_{1}^{-}$and the $\mathbf{E}^{-}$representations the energy of the ground state is much smaller than that of the exited state as one can see from the value of $\sqrt{s} / m_{\rho}$ in (2.1). Thus the energy of these 
states can be extracted by a single exponential fit for the time correlation functions of the $\rho$ meson. We use the local $\rho$ meson operator for the sink and a smearing operator for the source as discussed later.

For the $\mathbf{A}_{2}^{-}$representation the energy of the first exited state is close to that of the ground state. Thus we use the variational method [13] with a matrix of the time correlation function,

$$
G(t)=\left(\begin{array}{cc}
\left\langle 0\left|\Omega^{\dagger}(t) \bar{\Omega}\left(t_{s}\right)\right| 0\right\rangle & \left\langle 0\left|\Omega^{\dagger}(t) \overline{\rho_{3}}\left(t_{s}\right)\right| 0\right\rangle \\
\left\langle 0\left|\rho_{3}^{\dagger}(t) \bar{\Omega}\left(t_{s}\right)\right| 0\right\rangle & \left\langle 0\left|\rho_{3}^{\dagger}(t) \overline{\rho_{3}}\left(t_{s}\right)\right| 0\right\rangle
\end{array}\right) .
$$

We extract the energy by a single exponential fit for the two eigenvalues $\lambda_{1}(t)$ and $\lambda_{2}(t)$ of the matrix $M(t)=G(t) G^{-1}\left(t_{R}\right)$ with some reference time $t_{R}$, assuming that the lower two states dominate the correlation function. In (2.2), $\rho_{3}(t)$ is the local operator for the neutral $\rho$ meson at time $t$ with momentum $\mathbf{p}$ and the polarization vector parallel to the momentum. $\Omega(t)$ is an operator for the two pions with the momentum $\mathbf{0}$ and $\mathbf{p}=(2 \pi / L) \mathbf{e}_{3}$,

$$
\Omega(t)=\frac{1}{\sqrt{2}}\left(\pi^{+}\left(\mathbf{0}, t_{1}\right) \pi^{-}(\mathbf{p}, t)-\pi^{-}\left(\mathbf{0}, t_{1}\right) \pi^{+}(\mathbf{p}, t)\right) \times \mathrm{e}^{m_{\pi} \cdot\left(t_{1}-t\right)},
$$

where $\pi(\mathbf{p}, t)$ is the local pion operator with momentum $\mathbf{p}$ at time $t$. The times slice of the pion with zero momentum is fixed at $t_{1}>>t$, and an exponential time factor is introduced, so that the operator has the same time behavior as that of the usual Heisenberg operator for $t_{1}>>t$, ie., $\langle 0| \Omega^{\dagger}(t)=\langle 0| \Omega^{\dagger}(0) \exp (-H \cdot t)$.

Two operators $\overline{\rho_{3}}\left(t_{s}\right)$ and $\bar{\Omega}\left(t_{s}\right)$ are used for the source in (2.2), which are given by

$$
\begin{aligned}
& \bar{\Omega}\left(t_{s}\right)=\frac{1}{\sqrt{2}}\left(\pi^{+}\left(\mathbf{0}, t_{s}\right) \pi^{-}\left(\mathbf{p}, t_{s}\right)-\pi^{-}\left(\mathbf{0}, t_{s}\right) \pi^{+}\left(\mathbf{p}, t_{s}\right)\right), \\
& \overline{\rho_{3}}\left(t_{s}\right)=\sum_{\mathbf{z} \in \Gamma} \frac{1}{\sqrt{2}}\left(\bar{U}\left(\mathbf{z}, t_{s}\right) \gamma_{3} U\left(\mathbf{z}, t_{s}\right)-\bar{D}\left(\mathbf{z}, t_{s}\right) \gamma_{3} D\left(\mathbf{z}, t_{s}\right)\right) \mathrm{e}^{i \mathbf{p} \cdot \mathbf{z}} .
\end{aligned}
$$

The operator $U\left(\mathbf{z}, t_{s}\right)\left(D\left(\mathbf{z}, t_{s}\right)\right)$ is a smearing operator for the up (down) quark given by $U\left(\mathbf{z}, t_{s}\right)=$ $\sum_{\mathbf{x}} u\left(\mathbf{z}+\mathbf{x}, t_{s}\right) \cdot F(|\mathbf{x}-\mathbf{z}|)$, where $u\left(\mathbf{x}, t_{s}\right)$ is the quark operator at position $\mathbf{x}$ and time $t_{s}$. We use the same smearing function $F(x)$ as in Ref. [10]. This operator is used after fixing gauge configurations to the Coulomb gauge. In (2.5) a summation over $\mathbf{z}$ is taken to reduce contaminations from the states with different total momenta and $\Gamma=\left\{\mathbf{z} \mid \mathbf{z}=(L / 2) \cdot\left(n_{1}, n_{2}, n_{3}\right), n_{j}=0\right.$ or 1$\}$ is chosen in the present work. The smearing operator (2.5) is also used to extract the energy of the ground state for the $\mathbf{T}_{1}^{-}$and the $\mathbf{E}^{-}$representations setting the momentum to $\mathbf{p}=\mathbf{0}$ and $(2 \pi / L) \mathbf{e}_{3}$.

The quark contractions of $G(t)$ in (2.2) are given by

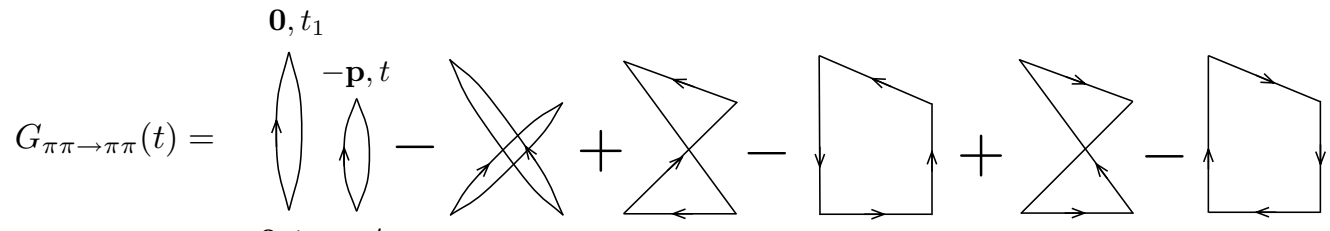

$$
\begin{aligned}
& \mathbf{0}, t_{s} \mathbf{p}, t_{s} \\
& G_{\pi \pi \rightarrow \rho}(t)=\bigwedge_{\mathbf{0}, t_{s}}^{-\mathbf{p}, t}-\bigwedge_{\mathbf{p}, t_{s}} G_{\rho \rightarrow \pi \pi}(t)=-\overbrace{\mathbf{p}, t_{s}}^{-\mathbf{p}, t}+
\end{aligned}
$$


where the time runs upward in the diagrams. The vertices refer to the pion or the $\rho$ meson operator with the momentum at the time specified in the diagrams. The $\rho$ meson operators at $t_{s}$ are the smearing operators, and the other is the local operator.

In order to calculate the quark contractions in (2.6), we use the source method and the stochastic noise method as in the previous work by CP-PACS Collaboration [7]. We introduce a $U(1)$ noise $\xi_{j}(\mathbf{x})$ which satisfies

$$
\sum_{j=1}^{N_{R}} \xi_{j}^{\dagger}(\mathbf{x}) \xi_{j}(\mathbf{y})=\delta^{3}(\mathbf{x}-\mathbf{y}) \quad \text { for } N_{R} \rightarrow \infty
$$

where $N_{R}$ is the number of noises taken to be 10 in the present work. We calculate the following four types of quark propagators :

$$
\begin{aligned}
& Q_{A B}\left(\mathbf{x}, t \mid \mathbf{q}, t_{s}, \xi_{j}\right)=\sum_{\mathbf{y}}\left(D^{-1}\right)_{A B}\left(\mathbf{x}, t ; \mathbf{y}, t_{s}\right) \cdot\left[\mathrm{e}^{i \mathbf{q} \cdot \mathbf{y}} \xi_{j}(\mathbf{y})\right] \\
& W_{A B}\left(\mathbf{x}, t\left|\mathbf{k}, t_{a}\right| \mathbf{q}, t_{s}, \xi_{j}\right)=\sum_{\mathbf{y}} \sum_{C}\left(D^{-1}\right)_{A C}\left(\mathbf{x}, t ; \mathbf{z}, t_{a}\right) \cdot\left[\mathrm{e}^{i \mathbf{k} \cdot \mathbf{z}} \gamma_{5} Q\left(\mathbf{z}, t_{a} \mid \mathbf{q}, t_{s}, \xi_{j}\right)\right]_{C B}, \\
& \bar{Q}_{A B}\left(\mathbf{x}, t \mid \mathbf{z}, t_{S}\right)=\sum_{\mathbf{y}}\left(D^{-1}\right)_{A B}\left(\mathbf{x}, t ; \mathbf{y}, t_{s}\right) \cdot[F(|\mathbf{y}-\mathbf{z}|)] \\
& \bar{W}_{A B}\left(\mathbf{x}, t\left|\mathbf{k}, t_{a}\right| \mathbf{z}, t_{s}\right)=\sum_{\mathbf{y}} \sum_{C}\left(D^{-1}\right)_{A C}\left(\mathbf{x}, t ; \mathbf{y}, t_{a}\right) \cdot\left[\mathrm{e}^{i \mathbf{k} \cdot \mathbf{y}} \gamma_{5} \bar{Q}\left(\mathbf{y}, t_{a} \mid \mathbf{z}, t_{s}\right)\right]_{C B},
\end{aligned}
$$

where $A$ and $B$ refer to color and spin indices, and $F(x)$ is the smearing function. The square bracket is used as the source for the inversion of the Dirac operator $D$.

The first term of the $\pi \pi \rightarrow \pi \pi$ component of (2.6) can be calculated by introducing an another $U(1)$ noise $\eta_{j}(\mathbf{x})$ having the same property as $\xi_{j}(\mathbf{x})$ in (2.7),

$$
\sum_{j} \sum_{\mathbf{x}, \mathbf{y}} \mathrm{e}^{-i \mathbf{p} \cdot \mathbf{y}} \cdot\left\langle Q^{\dagger}\left(\mathbf{x}, t_{1} \mid \mathbf{0}, t_{s}, \xi_{j}\right) Q\left(\mathbf{x}, t_{1} \mid \mathbf{0}, t_{s}, \xi_{j}\right)\right\rangle \cdot \mathrm{e}^{m_{\pi} \cdot\left(t_{1}-t\right)} \cdot\left\langle Q^{\dagger}\left(\mathbf{y}, t \mid \mathbf{p}, t_{s}, \eta_{j}\right) Q\left(\mathbf{y}, t \mid \mathbf{p}, t_{s}, \eta_{j}\right)\right\rangle,
$$

where the bracket means trace for the color and the spin indices. The exponential time factor comes from the definition of the operator of the two pions in (2.3). The second term is given by exchanging the momentum and the time slice of the sink in (2.12). The 3rd to 6th terms can be calculated by

$$
\begin{aligned}
& G_{\pi \pi \rightarrow \pi \pi}^{[3 \mathrm{rd}]}(t)=\sum_{j} \sum_{\mathbf{x}} \mathrm{e}^{-i \mathbf{p} \cdot \mathbf{x}} \cdot\left\langle W^{\dagger}\left(\mathbf{x}, t\left|\mathbf{0}, t_{1}\right| \mathbf{0}, t_{s}, \xi_{j}\right) W\left(\mathbf{x}, t\left|\mathbf{0}, t_{s}\right| \mathbf{p}, t_{s}, \xi_{j}\right)\right\rangle \cdot \mathrm{e}^{m_{\pi} \cdot\left(t_{1}-t\right)}, \\
& G_{\pi \pi \rightarrow \pi \pi}^{[4 \mathrm{th}]}(t)=\sum_{j} \sum_{\mathbf{x}} \mathrm{e}^{-i \mathbf{p} \cdot \mathbf{x}} \cdot\left\langle W^{\dagger}\left(\mathbf{x}, t\left|\mathbf{0}, t_{1}\right| \mathbf{0}, t_{s}, \xi_{j}\right) W\left(\mathbf{x}, t\left|\mathbf{p}, t_{s}\right| \mathbf{0}, t_{s}, \xi_{j}\right)\right\rangle \cdot \mathrm{e}^{m_{\pi} \cdot\left(t_{1}-t\right)}, \\
& G_{\pi \pi \rightarrow \pi \pi}^{[5 \mathrm{th}]}(t)=\sum_{j} \sum_{\mathbf{x}} \mathrm{e}^{-i \mathbf{p} \cdot \mathbf{x}} \cdot\left\langle W\left(\mathbf{x}, t\left|\mathbf{0}, t_{1}\right| \mathbf{0}, t_{s}, \xi_{j}\right) W^{\dagger}\left(\mathbf{x}, t\left|\mathbf{0}, t_{s}\right|-\mathbf{p}, t_{s}, \xi_{j}\right)\right\rangle \cdot \mathrm{e}^{m_{\pi} \cdot\left(t_{1}-t\right)}, \\
& G_{\pi \pi \rightarrow \pi \pi}^{[6 \mathrm{Gh}]}(t)=\sum_{j} \sum_{\mathbf{x}} \mathrm{e}^{-i \mathbf{p} \cdot \mathbf{x}} \cdot\left\langle W\left(\mathbf{x}, t\left|\mathbf{0}, t_{1}\right| \mathbf{0}, t_{s}, \xi_{j}\right) W^{\dagger}\left(\mathbf{x}, t\left|-\mathbf{p}, t_{s}\right| \mathbf{0}, t_{s}, \xi_{j}\right)\right\rangle \cdot \mathrm{e}^{m_{\pi} \cdot\left(t_{1}-t\right)}
\end{aligned}
$$

The two terms of $\pi \pi \rightarrow \rho$ of (2.6) can be similarly obtained by

$$
G_{\pi \pi \rightarrow \rho}^{[1 \mathrm{st}]}(t)=\sum_{j} \sum_{\mathbf{x}} \mathrm{e}^{-i \mathbf{p} \cdot \mathbf{x}} \cdot\left\langle W^{\dagger}\left(\mathbf{x}, t\left|-\mathbf{p}, t_{s}\right| \mathbf{0}, t_{s}, \xi_{j}\right)\left(\gamma_{5} \gamma_{3}\right) Q\left(\mathbf{x}, t \mid \mathbf{0}, t_{s}, \xi_{j}\right)\right\rangle
$$




$$
G_{\pi \pi \rightarrow \rho}^{[2 \mathrm{nd}]}(t)=\sum_{j} \sum_{\mathbf{x}} \mathrm{e}^{-i \mathbf{p} \cdot \mathbf{x}} \cdot\left\langle Q^{\dagger}\left(\mathbf{x}, t \mid \mathbf{0}, t_{s}, \xi_{j}\right)\left(\gamma_{5} \gamma_{3}\right) W\left(\mathbf{x}, t\left|\mathbf{p}, t_{s}\right| \mathbf{0}, t_{s}, \xi_{j}\right)\right\rangle .
$$

We can calculate the two terms of $\rho \rightarrow \pi \pi$ of (2.6) by

$$
\begin{aligned}
& G_{\rho \rightarrow \pi \pi}^{[1 \mathrm{st}]}(t)=\sum_{\mathbf{z} \in \Gamma} \mathrm{e}^{i \mathbf{p} \cdot \mathbf{z}} \sum_{\mathbf{x}} \mathrm{e}^{-i \mathbf{p} \cdot \mathbf{x}} \cdot\left\langle\bar{Q}^{\dagger}\left(\mathbf{x}, t \mid \mathbf{z}, t_{S}\right) \bar{W}\left(\mathbf{x}, t\left|\mathbf{0}, t_{1}\right| \mathbf{z}, t_{S}\right)\left(\gamma_{3} \gamma_{5}\right)\right\rangle \cdot \mathrm{e}^{m_{\pi} \cdot\left(t_{1}-t\right)} \\
& G_{\rho \rightarrow \pi \pi}^{[2 \mathrm{nd}]}(t)=\sum_{\mathbf{z} \in \Gamma} \mathrm{e}^{i \mathbf{p} \cdot \mathbf{z}} \sum_{\mathbf{x}} \mathrm{e}^{-i \mathbf{p} \cdot \mathbf{x}} \cdot\left\langle\bar{W}^{\dagger}\left(\mathbf{x}, t\left|\mathbf{0}, t_{1}\right| \mathbf{z}, t_{S}\right) \bar{Q}\left(\mathbf{x}, t \mid \mathbf{z}, t_{S}\right)\left(\gamma_{3} \gamma_{5}\right)\right\rangle \cdot \mathrm{e}^{m_{\pi} \cdot\left(t_{1}-t\right)}
\end{aligned}
$$

The $\rho \rightarrow \rho$ component is given by the $\bar{Q}$-type propagators as the usual two point function.

The quark propagators are calculated with the Dirichlet boundary condition imposed in the time direction and the source operators are set at $t_{s}=12$ to avoid effects from the temporal boundary. We put the zero momentum pion introduced in (2.3) at $t_{1}=42$. We calculate the $Q$-type propagators (2.8) for four combinations of $\mathbf{q}$ and $U(1)$ noise : (q, noise $)=\{(\mathbf{0}, \xi),(\mathbf{0}, \eta),(\mathbf{p}, \xi),(-\mathbf{p}, \xi)\}$. The $W$-type propagators (2.9) are calculated for five combinations of $\mathbf{k}, t_{a}$ and $\mathbf{q}:\left(\mathbf{k}, t_{a} \mid \mathbf{q}\right)=$ $\left\{\left(\mathbf{p}, t_{S} \mid \mathbf{0}\right),\left(-\mathbf{p}, t_{S} \mid \mathbf{0}\right),\left(\mathbf{0}, t_{S} \mid \mathbf{p}\right),\left(\mathbf{0}, t_{S} \mid-\mathbf{p}\right),\left(\mathbf{0}, t_{1} \mid \mathbf{0}\right)\right\}$, using the same $U(1)$ noise $\xi$ in common. We calculate the $\bar{Q}$-type propagator (2.10) and the $\bar{W}$-type propagator (2.11) with $\left(\mathbf{k}, t_{a}\right)=\left(\mathbf{0}, t_{1}\right)$ for the set $\mathbf{z} \in \Gamma$. Thus we calculate $(4+5) \times 10+(1+1) \times 8=106$ quark propagators for each configuration. The total number of the configurations analyzed every 10 trajectories is 440 . We estimate the statistical error by the jackknife method with bins of 400 trajectories.

\section{Results}

In the left panel of Fig. 1 we show the real part of the diagonal components ( $\pi \pi \rightarrow \pi \pi$ and $\rho \rightarrow \rho)$ and the imaginary part of the off-diagonal components $(\pi \pi \rightarrow \rho, \rho \rightarrow \pi \pi)$ of $G(t)$. The other real or imaginary part of the components vanish from $P$ and $C P$ symmetry. We calculate the two eigenvalues $\lambda_{n}(t)(n=1,2)$ for the matrix $M(t)=G(t) G^{-1}\left(t_{R}\right)$ with the reference time $t_{R}=23$. In the right panel of Fig. 1 we plot the eigenvalues normalized by the correlation function of the two free pions $N(t)=\left\langle 0\left|\pi(-\mathbf{p}, t) \pi\left(\mathbf{p}, t_{s}\right)\right| 0\right\rangle\left\langle 0\left|\pi(\mathbf{0}, t) \pi\left(\mathbf{0}, t_{s}\right)\right| 0\right\rangle$. Thus the slope of the figure corresponds to the energy difference with respect to the energy of the two free pions. We observe that the energy difference is negative for $\lambda_{1}(t)$ and positive for $\lambda_{2}(t)$. This means that the two-pion scatting phase shift is positive for the ground state and negative for the first exited state of the $\mathbf{A}_{2}^{-1}$ representation.

We extract the energies for both states by a single exponential fitting of the eigenvalues $\lambda_{n}(t)$ $(n=1,2)$ for the time range $t=24-33$. For the $\mathbf{T}_{1}^{-}$and the $\mathbf{E}^{-}$representations the energy of the ground state is obtained from the time correlation functions of the $\rho$ meson as explained before. Converting the energies on each frame to the invariant masses $\sqrt{s}$ and substituting them into the finite size formulas, we obtain the scattering phase shifts plotted in Fig. 2. In the figure we show $\left(k^{3} / \tan \delta(k)\right) / \sqrt{s}$ as function of $(\sqrt{s})^{2}$ in unit of the lattice cutoff, where $k=\sqrt{s / 4-m_{\pi}^{2}}$ is the scattering momentum.

In order to estimate the $\rho$ meson decay width at the physical quark mass we parametrize the scattering phase shift with the effective $\rho \rightarrow \pi \pi$ coupling constant $g_{\rho \pi \pi}$ by

$$
\frac{k^{3}}{\tan \delta(k)} / \sqrt{s}=\frac{6 \pi}{g_{\rho \pi \pi}^{2}} \cdot\left(M_{R}^{2}-s\right),
$$



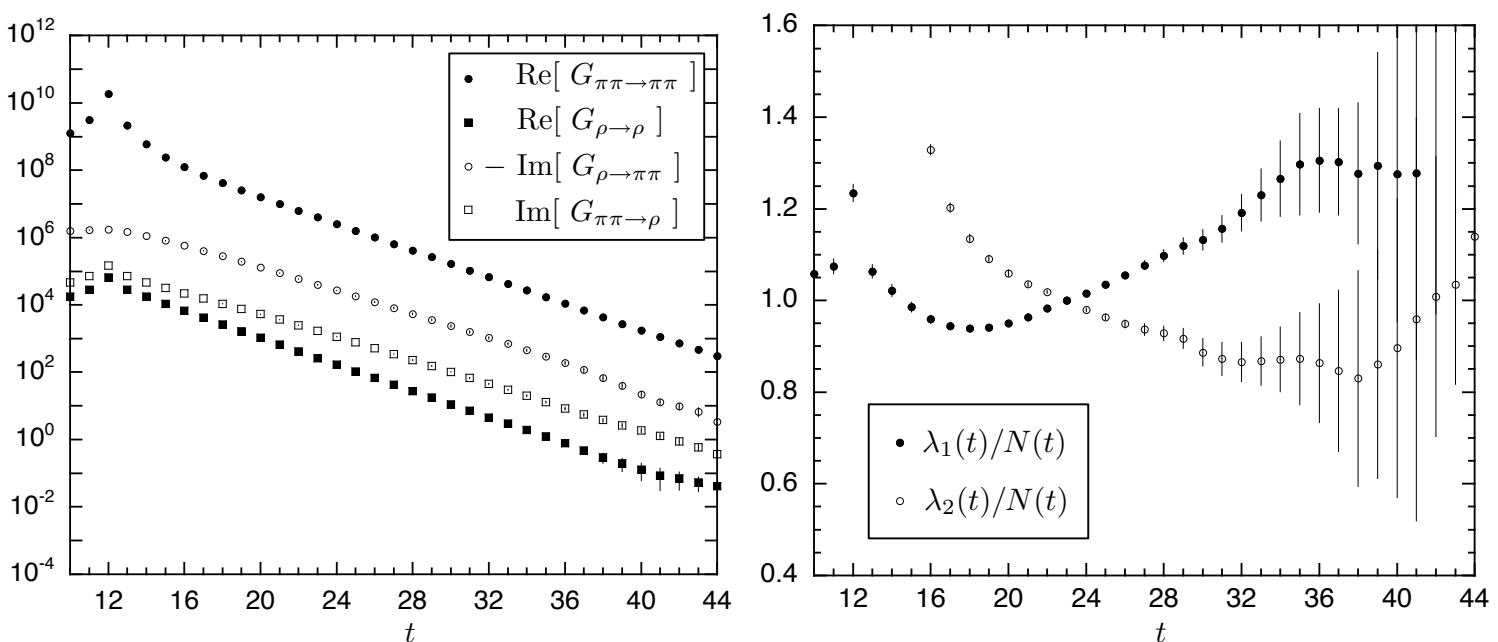

Figure 1: Left panel : Real part of the diagonal components $(\pi \pi \rightarrow \pi \pi$ and $\rho \rightarrow \rho)$ and imaginary part of the off-diagonal components $(\pi \pi \rightarrow \rho$ and $\rho \rightarrow \pi \pi$ ) of the time correlation function $G(t)$; Right panel : Eigenvalues $\lambda_{1}(t)$ and $\lambda_{2}(t)$ normalized by the correlation function of the two free pions $N(t)$.

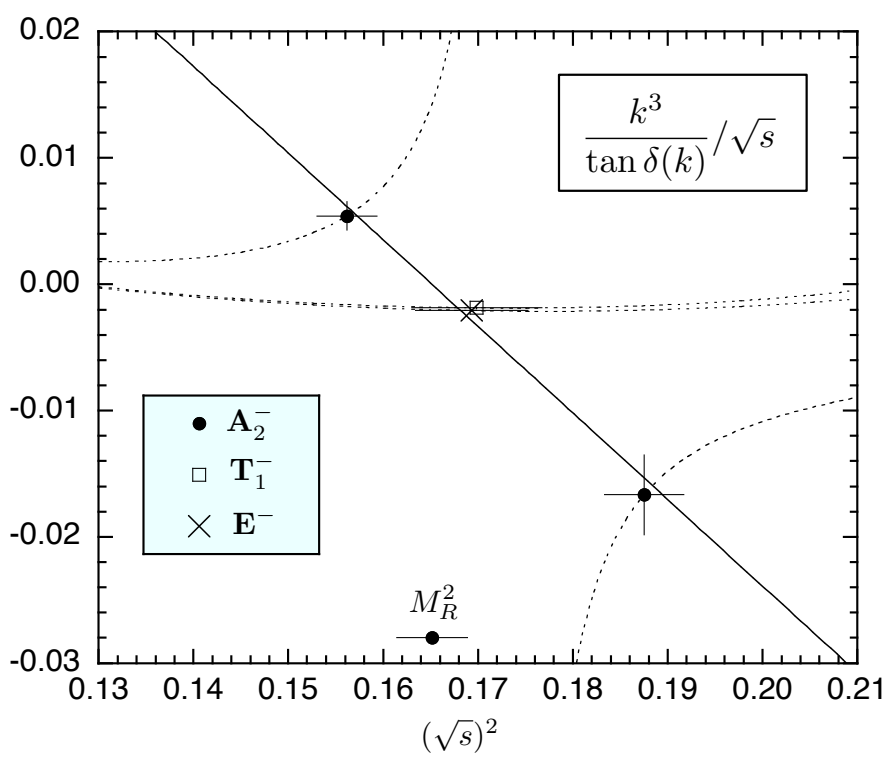

Figure 2: $\left(k^{3} / \tan \delta(k)\right) / \sqrt{s}$ as function of $(\sqrt{s})^{2}$ in unit of the lattice cutoff. Dotted lines refer to the finite size formulas for each representation.

where $M_{R}$ is the resonance mass. The coupling $g_{\rho \pi \pi}$ generally depends on the quark mass, but our present data at a single quark mass do not provide this information. Here we assume that the mass dependence is weak and try to estimate $g_{\rho \pi \pi}$ and $M_{R}$ by fitting our results with (3.1). We estimate the $\rho$ meson decay width at the physical quark mass from the formula $\Gamma_{\rho}=g_{\rho \pi \pi}^{2} \times 4.128 \mathrm{MeV}$. Our results of the fitting are given by

$$
a M_{R}=0.4064 \pm 0.046, \quad g_{\rho \pi \pi}=5.24 \pm 0.51, \quad \Gamma_{\rho}=113 \pm 22 \mathrm{MeV} .
$$

In Fig. 2 we indicate the result for the resonance mass $M_{R}$ and draw the fitting curve by a solid line. 
Our result of the $\rho$ meson decay width $\Gamma_{\rho}$ at the physical quark mass is smaller than the experimental value $(150 \mathrm{MeV})$. Possible reasons of the discrepancy are the mass dependence of the effective coupling constant $g_{\rho \pi \pi}$ and the finite lattice spacing effects. We will study these issues in next investigations.

\section{Summary}

In the present work we estimated the $\rho$ meson decay width from the $N_{f}=2+1$ full QCD configurations generated by PACS-CS Collaboration. The decay width is estimated from the $P$ wave scattering phase shift for $I=1$ two-pion system. We used the effective $\rho \rightarrow \pi \pi$ coupling constant $g_{\rho \pi \pi}$ to extrapolate from our simulation point $m_{\pi}=410 \mathrm{MeV}$ to the physical point $m_{\pi}=$ $135 \mathrm{MeV}$, assuming that $g_{\rho \pi \pi}$ does not depend on the quark mass. The decay width may be estimated directly from the energy dependence of the phase shift data assuming the Breit-Wigner resonance formula, if the simulations are made close to the physical quark mass and we have data at several values of energy near the resonance mass. We leave these issues to studies in the future.

This work is supported in part by Grants-in-Aid of the Ministry of Education (Nos. 20340047, 20105001, 20105003 , 20740139 , 20540248 , 21340049 , 22244018, 20105002 , $22105501,22740138,10143538,2110550120105005$ ). The numerical calculations have been carried out on PACS-CS at Center for Computational Sciences, University of Tsukuba.

\section{References}

[1] S. Gottlieb, P.B. Mackenzie, H.B. Thacker, and D. Weingarten, Phys. Lett. B134 (1984) 346.

[2] R.D. Loft and T.A. DeGrand, Phys. Rev. D39 (1989) 2692.

[3] UKQCD Collaboration, C. McNeile and C. Michael, Phys. Lett. B556 (2003) 177 [hep-lat/0212020].

[4] ETMC Collaboration, K. Jansen et al., Phys. Rev. D80 (2009) 054510 [arXiv:0906.4720 [hep-lat]].

[5] M. Lüscher, Commun. Math. Phys. 105 (1986) 153; Nucl. Phys. B354 (1991) 531.

[6] K. Rummukainen and S. Gottlieb, Nucl. Phys. B450 (1995) 397 [hep-lat/9503028].

[7] CP-PACS Collaboration, A. Aoki et al., Phys. Rev. D76 (2007) 094506 [arXiv:0708.3705 [hep-lat]].

[8] QCDSF Collaboration, M. Gockeler et al., PoS LATTICE2008 (2008) 136 [arXiv:0810.5337 [hep-lat]].

[9] ETMC collaboration, X. Feng, K. Jansena and D.B. Rennera, arXiv:0910.4871 [hep-lat].

[10] PACS-CS Collaboration, S. Aoki et al., Phys. Rev. D79 (2009) 034503 [arXiv:0807.1661 [hep-lat]].

[11] ETMC collaboration, X. Feng, K. Jansena and D.B. Rennera, these proceeding.

[12] BMW collaboration, J. Frison, these proceeding.

[13] M. Lüscher and U. Wolff, Nucl. Phys. B339 (1990) 222. 